\title{
Epstein-Barr Virus Nuclear Antigen-2 Detection and Typing in Immunocompromised Children Correlated With Lymphoproliferative Disorder Biopsy Findings
}

Thiago Marques Mendes, Léa Campos de Oliveira, Lídia Yamamoto, Gilda Maria Barbaro Del Negro and Thelma Suely Okay Laboratory of Medical Investigation (LIM 36), Department of Pediatrics, Medical School of São Paulo University; São Paulo, SP, Brazil

Epstein-Barr virus (EBV), the causative agent of infectious mononucleosis, plays a significant role as a cofactor in the process of tumorigenesis, and has consistently been associated with a variety of malignancies especially in immunocompromised patients. Forty-four children and adolescents (21 liver transplant patients, 7 heart transplant, 5 AIDS, 3 autoimmune hepatitis, 2 nephritic syndromes, 2 medullar aplasia, 2 primary immunodeficiency disorder patients, 1 thrombocytopenic purpura and 1 systemic lupus erythematosus) presenting with chronic active EBV infection (VCA-IgM persistently positive; VCA-IgG > $20 \mathrm{AU} / \mathrm{mL}$ and positive IgG - EBNA) had peripheral blood samples obtained during clinically characterized EBV reactivation episodes. DNA samples were amplified in order to detect and type EBV on the basis of the EBNA-2 sequence (EBNA2 protein is essential for EBV-driven immortalization of B lymphocytes). Although we have found a predominance of type 1 EBNA-2 virus (33/44; 75\%), 10 patients $(22.73 \%)$ carried type 2 EBNA-2, and one liver transplant patient $(2.27 \%)$ a mixture of the two types, the higher proportion of type $2 \mathrm{EBV}$, as well as the finding of one patient bearing the two types is in agreement with other reports held on lymphoproliferative disorder (LPD) patients, which analyzed tumor biopsies. We conclude that EBNA-2 detection and typing can be performed in peripheral blood samples, and the high prevalence of type 2 in our casuistic indicates that this population is actually at risk of developing LPD, and should be monitored. Key-Words: EBVA, EBNA-2, infectious mononucleosis, lymphoproliferative disorder, EBV genotyping,EBV-PCR.

Epstein-Barr virus (EBV), the causative agent of infectious mononucleosis, plays a significant role as a cofactor in the process of tumorigenesis, and has consistently been associated with a variety of malignancies including endemic Burkitt's lymphoma, nasopharyngeal carcinoma, certain B and T-cell lymphomas, approximately 50\% of Hodgkin's disease, different types of gastric carcinomas, and other lymphoproliferative diseases [1]. Immunosuppressive drugs such as cyclosporine A and tacrolimus seem to act synergistically with EBV as tumor inducing factors [2].

EBV infection of primary $B$ cells results in the establishment of a latent infection, in which only 11 of potentially more than 80 viral proteins are expressed. These gene products include EBV nuclear antigens EBNA 1, 2, 3A, 3B, 3C and 5, latent proteins (LMP) 1, 2A and 2B, and small non coding RNAs (EBER 1 and 2). The Epstein-Barr virus EBNA-2 protein is a transcriptional activator that regulates viral latent genes and cellular gene expression, and is also essential for EBV-driven immortalization of B lymphocytes $[3,4]$. EBNA-2 is critically required for B-cell transformation, as the P3HR1-derived EBV strain, which lacks EBNA-2, is deficient in this capacity, and the transforming potential is reconstituted with EBNA-2 expression [5,6].

EBV type 1 and type 2 (formerly called type A and B), appear to be mainly caused by divergence within the EBNA-

Received on 28 November 2007; revised 5 April 2008.

Address for correspondence: Dr. Thelma Suely Okay. Laboratório de Investigação Médica em Pediatria Clínica - LIM 36, Instituto da Criança - $5^{0}$ andar. Av. Dr. Enéas Carvalho de Aguiar, 647. Zip code: 05403900. Cerqueira César - São Paulo - SP, Brazil. Phone: (5511) 3069 8606. Fax : (5511) 3069 8591. E-mail: tsokay@icr.hcnet.usp.br.

The Brazilian Journal of Infectious Diseases
2 protein, because EBV type 2, when compared to type 1, has a lower transforming efficiency and a poorer initial outgrowth $[7,8]$. Both EBV types occur worldwide, with different geographical distributions. In general, EBV type 1 is the predominant type in lymphatic cells of immunocompetent, infected individuals. However, in certain T-cell deficient hosts, especially those with acquired immunodeficiency syndrome, there are increased infection rates with EBV type 2 [9,10].

Persistent infection with more than one EBV type or strain is not unusual, particularly in immunocompromised patients. Intertypic recombinants have been identified in the oropharynx and lymphocytes of immunodeficient patients, and less commonly from healthy people. Scientific evidence suggests that some immune impairment promotes co-infection with multiple strains of EBV, and fosters the development of intertypic recombinant viruses [10]. Human immunodeficiency virus type 1-infected patients develop a number of lymphoproliferative disorders ranging from non neoplastic lymphadenopathies to B-cell lymphomas [11]. Different point mutations in the EBNA-2 gene were described in some EBV type-1 isolates from immunodeficiency virus-infected patients, but not from immunodeficiency virus-negative subjects [12].

Although a large amount of in vitro scientific evidence concerning the differences of EBNA-2 type 1 and type 2 is available, little is known about whether both strains really differ in their biological activities in vivo. Aitken et al. [13] found EBNA-2 sequence heterogeneity in EBV type-1 isolates from African and New Guinean endemic Burkitt's lymphoma cases, suggesting that variant EBV type-1 sub-strains may be associated with these tumors.

Human EBV establishes latency and promotes the longterm survival of its host B cell by targeting the molecular machinery controlling cell fate decisions. EBNA-2 is a key determinant in the EBV-driven B-cell growth transformation 
process. By activating the array of viral and cellular target genes, EBNA-2 initiates a cascade of events which ultimately cause cell cycle entry and the proliferation of the infected B cell [14]. The mechanism of transcriptional activation by EBNA2 also involves phosphorylation of the C-terminal domain (CTD) of RNA polymerase II [15]. It was also established that somatic hypermutation and class-switch recombination in germinal centers critically depend on activation-induced cytidine deaminase (AID). Deregulation of AID may lead to the aberrant activation or persistence of both genetic processes, thus contributing to the pathogenesis of B-cell lymphomas by mistargeted mutagenesis or recombination [16]. The EBNA-2 also up-regulates bfl-1, an anti-apoptotic gene which confers protection from apoptosis under conditions of growth factor deprivation [17].

Taken into account that conventional diagnostic tools are rarely useful in evaluating EBV-related disorders in immunodeficient patients, polymerase chain reaction (PCR) offers the possibility of rapid and accurate EBV detection and typing in patients at risk of developing lymphoproliferative disorders, allowing a better understanding of the different pathogenic potentials of the two main viral types [18]. Another interesting point is to determine whether peripheral blood leukocytes could be used as the primary source of DNA instead of biopsy specimens, which would allow pediatricians to monitor immunocompromised patients with chronic-active infections by means of a less invasive procedure, as soon as EBV detection takes place. Therefore, we aimed at detecting and typing the EBV EBNA-2 gene in a group of immunocompromised children and adolescents by analyzing peripheral blood samples.

\section{Material and Methods}

This study was approved by the Ethics Committee of the "Faculdade de Medicina da Universidade de São Paulo", Brazil. After informed consent, we analyzed peripheral blood samples of 44 immunocompromised patients presenting with chronic-active EBV infection. These patients, 24 boys and 20 girls, from 5 to 14 years old, were assisted at the Children's Institute: 21 with liver transplants, 7 with heart transplants, 5 with AIDS, 3 with autoimmune hepatitis, 2 with nephritic syndromes, 2 with medullar aplasia, 2 with primary immunodeficiency disorders, 1 with thrombocytopenic purpura and 1 with systemic lupus erythematosus. All 44 cases were diagnosed as bearing chronic-active EBV infection on the basis of serological results: VCA-IgM persistently positive; VCA-IgG > $20 \mathrm{AU} / \mathrm{mL}$ and positive for IgG - EBNA. IgG antibodies to VCA were determined by capture ELISA (Diasorin, Italy); VCA-IgG positive $>20 \mathrm{AU} / \mathrm{mL}$ and VCAIgM positive (qualitative test); IgG to EBNA (nuclear antigen) was determined by ELISA (Sanofi Diagnostics Pasteur, France), samples being classified as acute EBV infection (positive for IgM and IgG to VCA but negative to IgG-EBNA), post-acute EBV infection or carrier state (positive for IgG to VCA and IgG-EBNA).

\section{DNA Extraction from Whole Blood}

Two milliliters of whole blood (EDTA, Becton Dickinson) was drawn from patients after informed consent of parents. DNA extraction was performed according to a previously described salting-out protocol [19]. DNA concentration was estimated by UV spectrophotometry at $260 \mathrm{~nm}$ (DU-70, Beckman).

\section{Amplification and Detection}

Before EBV specific amplifications, all DNA samples were tested with primers to amplify a fragment of the human betaactingene (sense 5'- GTGGGGCGCCCCAGGCACC-3' and antisense 5'-CTCCTTATT GTCACGCACGATTTC-3') in order to ensure DNA integrity and absence of amplification inhibitors. PCR assays were performed in $50 \mu \mathrm{L}$ of total volume containing approximately 100 ng genomic DNA, $100 \mathrm{mM}$ Tris$\mathrm{HCl}, 500 \mathrm{mM} \mathrm{KCl} ; 2.5$ units Taq DNA polymerase (Amersham, Biotech), $200 \mu \mathrm{M}$ dNTP (Invitrogen, USA), $1.5 \mathrm{mM} \mathrm{MgCl}_{2} ; 0.4$ $\mu \mathrm{M}$ each primer. In the first round of amplification, primers E2p1 and E2p2 aimed at amplifying a fragment of 596bp covering almost the entire EBNA2 gene extent (E2p1: 5'AGGGATGCCTGGACACAAGA-3' and E2p2: 5'TGGTGCTGCTGGTG GTGGCAAT-3'). In the second round of amplification, the pair of primers Ap1 and Ap2 aimed at amplifying a $497 \mathrm{bp}$ fragment characterizing the EBV type 1 EBNA2 gene product, while primers $\mathrm{Bp} 1$ and $\mathrm{Bp} 2$ amplified a 150 bp fragment specific to the EBV type 2 EBNA2 gene product (Ap1: 5'- TCTTGATAGGGA TCCGCTAGGATA-3'; Ap2: 5'-ACCGTGGTTCTGGACTATCTGGATC-3'; Bp1:5'CATGGTAGCCTTA GGACATA -3'; Bp2: 5'AGACTTAGTTGATGCCCTAG-3') [18]. All amplifications (beta-actin and EBV specific) were carried out as follows: after an initial denaturation step of $5 \mathrm{~min}$. at $95^{\circ} \mathrm{C}, 40$ cycles of amplification were performed in either the first, or the second round of amplifications (MJ Research, PT-150 minicycler). Cycles consisted of $1 \mathrm{~min}$. at $95^{\circ} \mathrm{C}, 1 \mathrm{~min}$. at $55^{\circ} \mathrm{C}$ and $1 \mathrm{~min}$. at $72^{\circ} \mathrm{C}$, followed by a final extension step of $5 \mathrm{~min}$. at $72^{\circ} \mathrm{C}$. Afterwards, $10 \mathrm{~mL}$ of amplification products were analyzed using horizontal electrophoresis (Horizon H-58, Life Technologies, USA), in 2\% ethidium bromide-stained agarose gel (Sigma, USA). In each experiment, a negative control containing sterile water instead of genomic DNA, or DNA from a non infected individual was tested, as well as a positive control (EBV DNA from viral cultures).

\section{Results}

All 44 DNA samples were successfully amplified by the human beta-actin primers thus confirming integrity of DNA samples, and the absence of PCR inhibitors. The first round of amplification was followed by the second PCR with EBNA2 type 1 and type 2 specific primers (Figure 1). From a total of 44 samples, there were 33 type 1 EBNA-2 virus (33/44 or $75.0 \%$ ), 10 type 2 (10/44 or $22.73 \%$ ), and one DNA sample belonging to a liver transplant recipient patient which amplified type 1 and type 2 at the same time ( $01 / 44$ or $2.27 \%$ ). Table 1 summarizes PCR detection and typing and the patients' underlying disease. 
Figure 1. Electrophoresis of the nested-PCR products. A- First round (external primers E2p1 and E2p2). M - molecular weight marker - 100 bp (Amersham); lanes 1A, 3A, 5A and 7A - negative controls (sterile water instead of DNA); lane 2A- patient 1, lane 4A- patient 2; lane 6A- positive control (EBV-DNA). B- Second round (EBV type 1 and 2 specific primers, Ap1-2 and Bp1-2, respectively). $\mathrm{M}$ - molecular weight marker - $100 \mathrm{bp}$ (Amersham); lane 1B - negative control (sterile water instead of DNA); lanes 2B and 3B - Patient 1; lanes 4B and 5B - Patient 2; lane 6B - positive control (EBV type 1); lane 7A- positive control (EBV type 2).

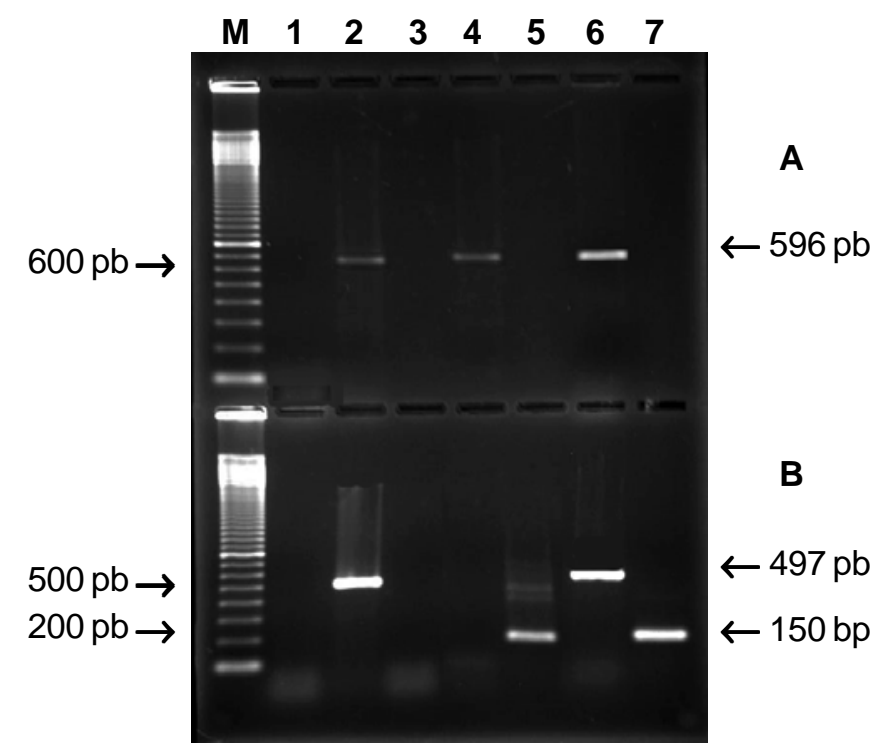

Table 1. Description of patients according to the underlying disease and the EBNA-2 typing by PCR.

\begin{tabular}{lcccc}
\hline Disease & N & Type 1 & Type 2 & Type 1 and 2 \\
\hline Liver transplantation & 21 & 15 & 5 & 1 \\
Heart transplantation & 7 & 6 & 1 & 0 \\
AIDS & 5 & 2 & 3 & 0 \\
Autoimmune hepatitis & 3 & 3 & 0 & 0 \\
Nephritic syndrome & 2 & 2 & 0 & 0 \\
Medullar aplasia & 2 & 2 & 0 & 0 \\
Primary immunodeficiency & 2 & 2 & 0 & 0 \\
Thrombocytopenic purpura & 1 & 1 & 0 & 0 \\
SLE & 1 & 0 & 1 & 0 \\
\hline Total & $\mathbf{4 4}$ & $\mathbf{3 3}$ & $\mathbf{1 0}$ & $\mathbf{1}$ \\
\hline
\end{tabular}

\section{Discussion}

In Brazil there are three reports on the detection and typing of EBV in pediatric patients. Araújo et al. [20] found type 1 EBV in the majority of Brazilian Burkitt's lymphoma cases: 20/ 30 type 1, 7/30 type 2 and 3 samples were not amplifiable. More recently [21], the same group studied the phenotype and the expression of EBV gene products in 90 classical Hodgkin and non Hodgkin's disease pediatric cases by immunohistochemistry, and in situ hybridization. They found that EBV infection was present in $86.7 \%$ of patients, but exclusively in classical Hodgkin's disease biopsies, although associated with all EBV subtypes. Hassan et al. [22] compared an EBV-RNA(EBER) in situ hybridization assay with a nested-
PCR that aimed at typing the EBNA-2 gene in 41 children presenting with B-cell non Hodgkin's lymphoma, including 35 Burkitt's lymphomas. They successfully detected EBV genomes in $68 \%$ of biopsies, and type 1 and type 2 EBV accounted for 80 and $20 \%$ of the samples, respectively. PCR and in situ hybridization were concordant in 95\% of results.

We studied 44 children and adolescents presenting with severe underlying diseases and receiving immunosuppressive therapy. However, we did not investigate any patient with overt lymphoproliferative disorders. Nevertheless, our findings corroborate those of Hassan et al. [22] as we found very similar distribution of type 1 and 2 EBNA-2: 75\% of type 1; $22.73 \%$ of type 2 and $2.27 \%$ of simultaneous infection (type 1 and type 2). 
Norin et al. [23] studied the incidence of lymphoproliferative disorders (LPD), in a series of 500 liver transplantations. They found that the rate varied from 1 to $10 \%$ depending on the type of organ transplanted, and the immunosuppressive regimens used. They concluded that post transplant LPD constitute a significant cause of complication. In the present study, there was a predominance of type 1 EBNA-2 EBV among the 21 liver transplant patients studied (15/21 or $71.43 \%$ ), with type 2 found in $5 / 21$ patients (23.81\%), and both types in one patient (1/21 or 4.76\%). Whether EBV typing will be useful in preventing, and promoting early diagnosis of LPD in these patients has yet to be verified.

EBV causes a wide spectrum of diseases in the setting of immunodeficiency including primary or secondary (acquired) immunodeficiencies, which have been increasingly reported [24]. The major clinical phenotype is the EBV genome-positive LPD, which ranges from benign lymphoproliferation to malignant lymphoma with chromosome alterations. Severe or fatal infectious mononucleosis may develop in some patients with immunodeficiency disorders such as X-linked diseases. Canioni et al. [25] evaluated distinctive features of LPD in a group of 18 primary immunodeficiency patients and compared the findings with those of 10 post transplant children. They concluded that LPD is much more pleomorphic in primary immunodeficiency patients compared to post transplant recipients, even if some LPD are similar in both groups. A low T-cell count and abnormal T-cell function indicated bad prognosis in both groups. Polymorphic LPD were most frequent $(n=19)$, whereas lymphomas were rare $(n=7)$, and pseudo-tumoral lymphoid hyperplasia $(\mathrm{n}=2)$ was only observed in the group of primary immunodeficiency patients. Several reports have demonstrated that the responses of Bcells to the Epstein-Barr virus are highly heterogeneous in common variable immunodeficiency. Kondo et al. [26] have studied patients with selected primary immunodeficiency disorders (Bloom's syndrome, Wiskott-Aldrich Syndrome, IgA deficiency). In Bloom's Syndrome, a mild B-cell function abnormality was found, while in the Wiskott-Aldrich's patients responses were variable. In a patient with IgA deficiency, peripheral blood mononuclear cells responded adequately to EBV in terms of proliferation, but poorly in IgA production, suggesting an abnormality only in the IgA production mechanism. Unfortunately, none of the mentioned studies have determined EBV type 1 and type 2 prevalence. In our casuistic, we have included only two patients with primary autoimmune deficiency, and in both cases the classical EBNA-2 type 1 was found. EBNA-2 and LMP1 gene analysis of 48 LPD from HIV1 patients evidenced EBNA-2 transcripts in all tumors, $50 \%$ of samples were co-infected with both EBV type 1 and 2 strains and/or a multitude of type 1 variants, with predominance of type 1 in large cell lymphomas, and type 2 in Burkitt-like tumors [11]. In our study, the five AIDS patients included did not present with LPD, but were undeniably at-risk patients. Although we observed a predominance of type 2 virus (2 type 1 , and 3 type 2 EBNA-2), further studies with more AIDS patients are needed to confirm this finding. Furthermore, we did not find any AIDS patient presenting with both EBV types. Kanegane et al. [27] have associated cases of acute idiopathic thrombocytopenic purpura with primary EBV infections. More recently, Yenicesu et al. [28] conducted a retrospective study based on the medical notes of pediatric patients with idiopathic thrombocytopenic purpura in whom EBV, CMV and rubella virus serology were performed, and viral infection was detected in $13.3 \%$ of cases. These studies did not analyze the EBV infection at the molecular level. In our study group, only one patient with idiopathic thrombocytopenic purpura was included, and had a type $1 \mathrm{EBV}$ virus.

EBV infection can cause a number of renal disorders ranging from microscopic hematuria, to acute renal failure. Membranous nephropathy is an uncommon and usually secondary cause of nephritic syndrome in children, and has been reported after chronic EBV infections with persistent antigenemia. Araya et al. [29] reported two pediatric cases of secondary membranous nephropathy associated with acute and chronic EBV infection. The association of EBV with renal histological changes consistent with membranous nephropathy has been previously suggested, but not directly described. In the study performed by Araya et al. [29] EBV infections were not detected and typed by PCR, so that they cannot state what type of EBV was at the origin of infections. In our study, the two patients with nephritic syndrome analyzed had type 1 EBNA-2 virus. In addition, to examine the possible involvement of EBV in the etiology of renal cell carcinoma (RCC), 9 RCC, 2 nephroblastoma (Wilm's tumor), and 2 RCC cell lines were subjected to mRNA in situ hybridization and indirect immunofluorescence staining. It was found that EBV infected all the RCC and nephroblastoma patients irrespective of the histological or clinical stage. The results also suggest that EBV expression may be involved in the pathogenesis of RCC and nephroblastoma [30].

To investigate the possibility that Epstein-Barr virus might play a role in systemic lupus erythematosus (SLE), EBV was searched for by PCR and by culture isolation in oropharyngeal lavage fluids of 15 SLE patients, of 13 acute infectious mononucleosis patients, and of 28 healthy individuals. EBV type 1 DNA was demonstrated by PCR in the oropharyngeal secretions of 8 SLE patients, and the virus was isolated from 6 DNA-positive specimens. Moreover, 50\% of the SLE patients, and $100 \%$ of the infectious mononucleosis patients, but none of the EBV-seropositive normal individuals, produced IgG antibodies raised against synthetic EBNA-2 peptides used in the study. The authors concluded that EBV may establish a persistent infection, at least in a certain number of SLE patients [31]. We have analyzed only one DNA sample from a SLE patient undergoing prolonged immunosuppressive therapy, and experiencing an EBV reactivation episode at the time of blood sampling. The nested-PCR to type the EBNA-2 gene found a type 2 virus in this patient. Again, it is premature to affirm the predominance of a certain subtype of EBV based on one SLE case, but it is important to emphasize that in the 
totality of patients included in the present study, it was possible to detect and type EBV using DNA extracted from peripheral blood samples drained during clinically characterized reactivation episodes.

Our nested-PCR technique has successfully detected and typed all 44 samples included in the present study probably because all patients were correctly diagnosed as having chronic-active EBV infection, and the samples were drawn from patients during reactivation episodes. Kunimoto et al. [32] have used a one-step amplification targeting the gene encoding the EBNA-2 nuclear antigen. EBV was detected in mouth washings of 21 (23\%) out of 91 seropositive healthy adults. Twenty samples (22\%) contained type 1 , and only one (1\%) type 2. Seventy-nine patients with various types of tonsillitis were also studied. EBV was detected in mouth washings of 37 patients (47\%): 34 (43\%) were type 1, and 3 (4\%) were type 2 . Double infection was not seen in either group. They concluded that EBV type 1 was the dominant type in Japan, and that the type 2 variant was quite rare. More recently, Peh et al. [33] aimed at comparing the performances of a one-step and a nested-PCR to detect the EBNA-2 gene. They studied 38 paraffin-embedded tissue lymphomas and 14 nasopharyngeal carcinomas, along with 12 reactive lymph nodes and tonsils as normal controls. All cases showed presence of type 1 (called type A) virus, consistently detected by nested-PCR, but not with the one-step amplification. They did not find any type 2 (called type B) samples, or mixed infection in the casuistic. They concluded that the nestedPCR technique used had successfully increased the sensitivity of EBV detection and typing, and type 1 EBV was the prevalent strain associated with human diseases in Malaysia.

In our casuistic we found a predominance of type 1 EBNA2 virus among the 44 samples studied, corresponding to $75.0 \%$ of the total. However, $22.73 \%$ of samples carried type 2 EBNA2 virus, and $2.27 \%$ a mixture of the two types. The fact that the casuistic was composed of immunocompromised children and adolescents could account for the higher proportion of type 2 EBV with respect to studies conducted in healthy populations. When these results are compared with those of LPD bearing patients, the prevalence of type 1 , type 2 , and both types, are similar, indicating that our population is actually at risk of developing LPD.

Strategies for the prophylaxis and treatment of these potentially life-threatening complications of EBV infection have advanced dramatically. They include immunologicalbased approaches targeted at EBV-infected cells, as well as improvement in the treatment of the underlying and predisposing diseases [34]. EBV detection and typing might also contribute to these strategies at a diagnostic and prognostic level.

\section{References}

1. McClain K.L. Epstein-Barr virus and HIV-associated diseases. Biomed Pharmacother 2001;55(7):348-52.
2. Chand D.H., Southerland S.M., Cunningham R.J. 3rd. Tacrolimus: the good, the bad and the ugly. Pediatr Transplant 2001;5(1):32-6.

3. Fuentes-Pananá E., Ling P.D. Characterization of the CBF2 binding site within the Epstein-Barr virus latency $\mathrm{C}$ promoter and its role in modulating EBNA2-mediated transactivation. Journal of Virology 1998;72(1):693-700.

4. Grabusic K., Maier S., Hartmann A., et al. The CR4 region of EBNA-2 confers viability of Epstein-Barr virus-transformed B cells by CBF-1-independent signaling. J Gen Virol 2006; 87(11): 3169-3176.

5. Cohen JL, Wang F, Mannick J, et al. Epstein-Barr virus nuclear protein 2 is a key determinant of lymphocyte transformation. Proc Natl Acad Sci USA 1989;86:9558-62.

6. Rabson M., Gradoville L., Heston L., et al. Non-immortalizing P3J-HR-1 Epstein-Barr virus: a deletion mutant of its transforming parent, Jijoye. J Virol 1982;44:834-44.

7. Sculley T.B., Sculley D.G., Pope J.H., et al. Epstein-Barr virus nuclear antigens 1 and 2 in Burkitt lymphoma cell lines containing either A or B - type virus. Intervirology 1988;29(2):77-85.

8. Rickinson A.B., Young L.S., Rowe M. Influence of the Epsteinbarr virus nuclear antigen EBNA-2 on the growth phenotype of virus-transformed B cells. J Virol 1987;61:1310-7.

9. Borisch B., Finke J., Hennig I., et al. Distribution and localization of Epstein-Barr virus subtypes A and B in AIDS-related lymphomas and lymphatic tissue of HIV-positive patients. J Pathol 1992;168:229-36.

10. Aguirre A.J., Robertson E.S. Characterization of intertypic recombinants of the Epstein-Barr virus from the body-cavitybased lymphomas cell lines BC-1 and BC-2. Virology 1999;264(2):359-69.

11. Ometto L., Menin C., Masiero S., et al. Molecular profile of Eptein- Barr Virus in Human Immunodeficiency Virus Type 1related lymphadenopathies and lymphomas. Blood 1997;90(1):313-22.

12. Buisson M., Morand P., Genoulaz O., et al. Changes in the dominant Epstein-Barr virus type during human immunodeficiency virus infection. J Gen Virol 1994;75:431-7.

13. Aitken C., Sengupta S.K., Aedes C., et al. Heterogeneity within the Epstein-Barr virus Nuclear Antigen 2 gene in different strains of Epstein-Barr virus. J Gen Virol 1994;75:95.

14. Maier S., Staffler G., Hartmann A., et al. Cellular target genes of Epstein-Barr virus nuclear antigen 2. J Virol 2006;80(19):9761-71.

15. Bark-Jones S.J., Webb H.M., West M.J. EBV EBNA-2 stimulates CDK-9-dependent transcription and RNA polymerase II phosphorylation on serine 5. Oncogene 2006;25(12):1775-85.

16. Tobollik S., Meyer L., Buettner M., et al. Epstein-Barr virus nuclear antigen 2 inhibits AID expression during EBV-driven B-cell growth. Blood 2006;108(12):3859-64.

17. Pegman P.M., Smith S.M., D’Souza B.N., et al. Epstein-Barr virus nuclear antigen 2 trans-activates the cellular antiapoptotic bfl1 gene by a CBF-1/RBJ kappa-dependent pathway. J Virol 2006;80(16):8133-44.

18. Telenti A., Marshall W.F., Smith T.F. Detection of Epstein-Barr virus by polymerase chain reaction. J Clin Microbiol 1990;28(10):2187-90.

19. Miller A.S., Dykes D.D., Polensky H.F. A simple salting out procedure for extracting DNA from human nucleated cells. Nucleic Acids Res 1988; $16: 1215$.

20. Araujo I., Foss H.-D., Bittencourt A., et al. Expression of EpsteinBarr vírus gene products in Burkitt's lymphoma in Northeast Brazil. Blood 1996;87(12):5279-86.

21. Araujo I., Bittencourt A.L., Barbosa H.S., et al. The high frequency of EBV infection in pediatric Hodgkin Lymphoma is related to the classical type in Bahia, Brazil. Virchows Arch 2006;449(3):315-9. 
22. Hassan R., White L.R., Stefanoff C.G., et al. Epstein-Barr virus (EBV) detection and typing by PCR: a contribution to diagnostic screening of EBV-positive Burkitt's lymphoma. Diagn Pathol 2006; $1: 1-7$.

23. Norin S., Kimby E., Ericzon B.G., et al. Posttransplant lymphoma: a single-center experience of 500 liver transplantation. Med Oncol 2004;21(3):273-84.

24. Okano M. Epstein-Barr virus in patients with immunodeficiency disorders. Biomed Pharmacother 2001;55(7):353-61.

25. Canioni D., Jabado N., MacIntyre E., et al. Lymphoproliferative disorders in children with primary immunodeficiencies: immunological status may be more predictive of the outcome than other criteria. Histopathology 2001;38(2):146-59.

26. Kondo N., Inoue R., Orii T. Responses of lymphocytes to EpsteinBarr vírus in patients with primary immunodeficiencies. J Investig Allergol Clin Immunol 1994;4(4):182-4.

27. Kanegane H., Miyawaki T., Iwai K., et al. Acute Thrombocytopenic Purpura associated with primary Epstein-Barr virus infection. Acta Pediatr Jpn 1994;36(4):423-6.

28. Yenicesu I., Yetgin S., Ozyürek E., et al. Virus-associated immune thrombocytopenic purpura in childhood. Pediatr Hematol Oncol 2002;19(6):433-7.
29. Araya C.E., González-Peralta R.P., Skoda-Smith S., et al. Systemic Epstein-Barr virus infection associated with membranous nephropathy in children. Clin Nephrol 2006;65(3):160-4.

30. Shimage M., Kawahara K., Harada S., et al. Expression of Epstein-Barr virus in renal cell carcinoma. Oncol Rep 2007;18(1):41-6.

31. Incaprera M., Rindi L., Bazzichi A., et al. Potential role of Epstein-Barr virus in systemic lupus erythematosus autoimunity. Clin Exp Rheumatol $1998 ; 16(3): 289-94$.

32. Kunimoto M., Tamura S., Tabata T., et al. One-step typing of Epstein-Barr vírus by polymerase chain reaction: predominance of type 1 virus in Japan. J Gen Virol 1992; 73:455-61.

33. Peh S.C., Kim L.H., Poppema S. Frequent presence of subtype A virus in Epstein-Barr virus-associated malignancies. Pathology 2002;34(5):446-50.

34. Okano M., Gross T.G. Advanced therapeutic and prophylactic strategies for Epstein-Barr virus infection in immunocompromised patients. Expert Rev Anti Infect Ther 2007;5(3):403-13. 